\title{
Amorphous Solid Phase Deposition of Ions and Phosphate within Eukaryotic Mitochondrial Matrices - Imaging and Characterization by CryoSTEM Tomography and Energy- Dispersive X-ray Spectroscopy
}

\author{
Sharon G. Wolf ${ }^{1}$, Yael Mutsafi ${ }^{2}$, Tal Ilani ${ }^{2}$, Michael Elbaum ${ }^{3}$ and Deborah Fass ${ }^{2}$ \\ 1. Department of Chemical Research Support, Weizmann Institute of Science, Rehovot Israel. \\ 2. Department of Structural Biology, Weizmann Institute of Science, Rehovot Israel. \\ 3. Department of Materials and Interfaces, Weizmann Institute of Science, Rehovot Israel.
}

CryoSTEM tomography (CSTET) is a novel combination ${ }^{1-4}$ of two well-established techniques: cryoelectron tomography (CET) and scanning transmission electron microscopy (STEM). The best current method for faithful preservation of cells is provided by cryogenic fixation. CET of such cryo-preserved prokaryotic and eukaryotic cells has provided rich detail on the in-situ organization of macromolecules and organelles ${ }^{5}$. However, sample thickness is limited to around $300 \mathrm{~nm}$ (close to the mean free path for inelastic scattering), due to the dependence on phase contrast from elastically scattered electrons. Using energy filters, inelastically scattered electrons can be blocked off, but this reduces signal at higher tilts while still depositing damaging energy into the sample. In addition, correction of the contrast transfer function at high tilts is not straightforward.

STEM provides a possibility for circumventing these issues because signal is acquired incoherently, with contrast provided by variations in mass thickness along the path of the electron, and atomic number. The natural limit for sample thickness is extended to the realm of the mean free path for elastic scattering, which is roughly three times that for inelastic scattering for biological and soft materials specimens consisting of light elements ${ }^{4}$. In addition, the STEM data acquisition naturally allows for dynamic focusing, providing fully focused images even at high tilts. Key elements for successful data collection include careful choice of semi-convergence angle and distribution of electron dose.

While the bright-field STEM data provides excellent morphological information, the simultaneously collected dark field signal provides information on chemical content, due to its sensitivity to atomic number. In addition, Energy-dispersive X-ray spectroscopy (EDX) and and electron energy loss spectroscopy (EELS) analysis can provide on-the-spot chemical characterization.

Using these methods, we have studied amorphous deposits of calcium phosphate granules in nonmineralizing eukaryotic vitrified and unsectioned cells. These so-called "dense granules" have been studied in isolated mitochondria ${ }^{6}$ but solid-phase ion storage in mitochondria within intact cells has not been well characterized. In the context of bone formation, mitochondria have been proposed to transport calcium phosphate to the cell surface ${ }^{7}$. We show that phosphate and calcium storage in mitochondria is a widespread phenomenon and may occur for purposes other than export for mineralization.

\section{References:}

[1] SG Wolf, L Houben and M Elbaum, Nature Methods 11(4) (2014), 423-428.

[2] SG Wolf, P Rez and M Elbaum, J. Microsc. 260(2) (2015), 227-233.

[3] M Elbaum, SG Wolf and L Houben, MRS Bulletin 41(7) (2016), 542-548.

[4] P Rez, T Larsen and M Elbaum, J. Struct.Biol. 196(3) (2016), 466-478. 
[5] M Beck and W Baumeister, Trends Cell Biol. 26(11) (2016), 825-837.

[6] AL Lehninger, Biochem. J. 119 (1970), 129-138.

[7] S Boonrungsiman et al, PNAS 109 (2012), 14170-14175.

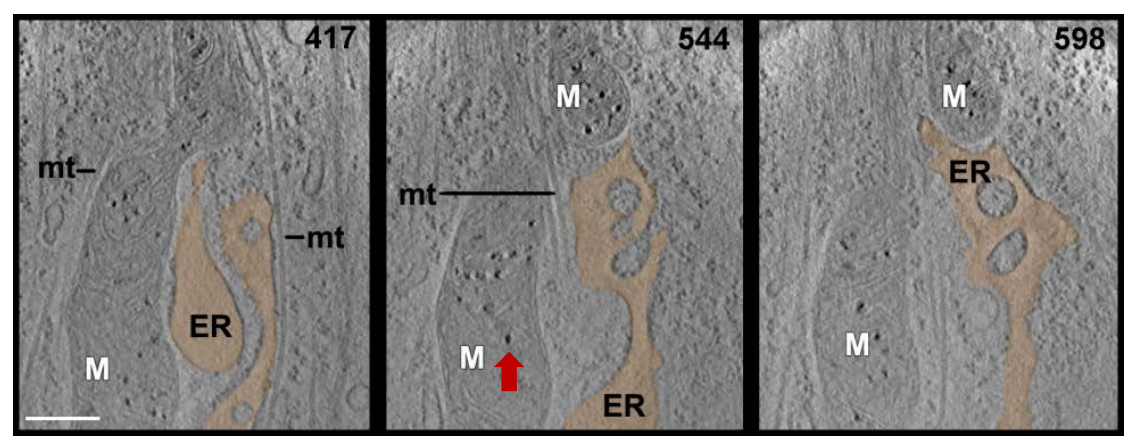

Figure 1. Cryo-STEM tomography reveals ion stores in intact mammalian cells. Three 30-nm thick sections from 3D tomographic reconstruction of an intact fibroblast cell reveal a mitochondrial-ER junction. Mitochondria contain numerous dense granules in the matrix (red arrow). M-mitochondrion, ER-endoplasmic reticulum (false-colored), mt-microtubules. Numbers in upper right indicate height in $\mathrm{nm}$ from the bottom of the cell.

Scale bar $=400 \mathrm{~nm}$. Total cell thickness $700 \mathrm{~nm}$.

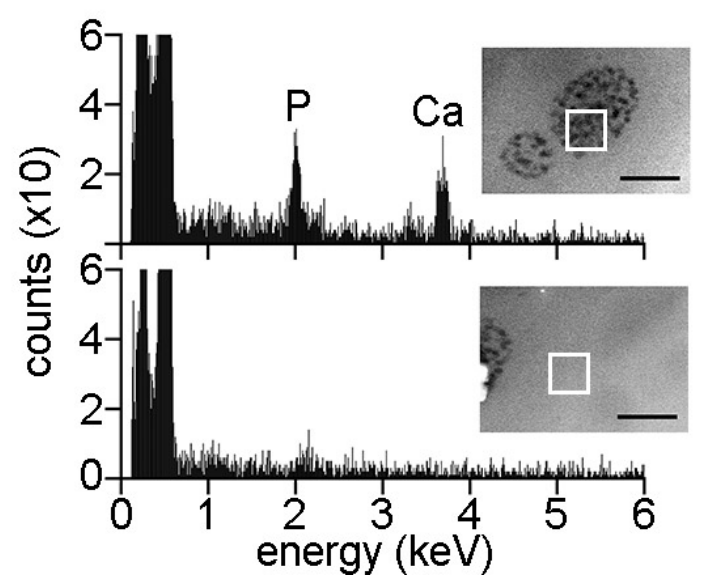

Figure 2. Mitochondrial granules contain amorphous calcium phosphate. EDX identifies calcium and phosphorus enrichment in mitochondria. Granules show no evidence of crystallinity. Scale bars $=500$ nm. 